\title{
MICROWAVE EMISSION FROM A SUNSPOT III. IMPLICATIONS FOR THE FORCE BALANCE IN A STATIC SUNSPOT
}

\author{
JEONGWOO W. LEE, DALE E. GARY, G. J. HURFORD, \\ and H. ZIRIN \\ Solar Astronomy 264-33, California Institute of Technology \\ Pasadena, CA 91125, U.S.A.
}

\begin{abstract}
We present our earlier measurement of the coronal fields above a sunspot in the active region, NOAA \#4741, which was obtained using microwave spectroscopy from the Owens Valley Radio Observatory (OVRO) in a form that allows comparison with theoretical models. Our empirical results for the coronal fields are compared with several theoretical models in order to understand the magnetic structure of a static sunspot. It is found that the presence of transverse pressure gradients should be considered to get a satisfactory fit.
\end{abstract}

\section{INTRODUCTION}

The magnetic field structure of the active region corona has been a popular subject in microwave studies of solar active regions, as the solar microwave radiation is known to provide a unique opportunity to detect the coronal fields directly (e.g., Alissandrakis, Kundu, \& Lantos 1980). One technique to measure the coronal fields is to make use of the thermal gyroresonance spectrum. This method has been discussed and implemented by Hurford \& Gary (1986) (see also Gelfreikh $t t$ al. presented in this proceedings). In Papers I and II (Lee, Hurford, \& Gary 1992 and Lee, Gary, \& Hurford 1992, respectively), we applied Hurford \& Gary's (1986) procedure to derive the coronal field distribution in the active region NOAA \#4741 along both the vertical and the horizontal directions. This was possible because we had multi-frequency, interferometric observations at OVRO at both the disk center and the limb. Since these results are new and entirely empirical, it is of new interest to test whether the coronal field distribution inferred from our observations can be reproduced by any simple theoretical model. In this paper, we make a comparison of our observational results with the dipole model, the solenoid model of Sakurai \& Uchida (1977), and the hydrostatic equilibrium model of Low (1980) to yield an implication for the static magnetic structure of a sunspot. In $\S 2$, the results of our sunspot field measurement are briefly presented and compared with theoretical models. Implications for the force balance in a sunspot are discussed in $§ 3$. Conclusion will follow in $\$ 4$. 


\section{OBSERVED MAGNETIC FIELDS us THEORETICAL MODELS}

For the comparison with theoretical models, we use the cylindrical coordinates where $\hat{z}$ is along the symmetry axis of the spot and $z=0$ is at the waist of the spot field lines. In these coordinates, our results reported in Papers I and II can be rewritten as

$$
\begin{gathered}
B(r, z=d+H)=1420(1 \pm 0.08) \exp \left[-\left(\frac{r}{11.05^{\prime \prime} \pm 0.16^{\prime \prime}}\right)^{2}\right] \mathrm{G}, \\
B(r=0, z=d+H+h)=860(1 \pm 0.03) \mathrm{G} \\
\text { and } B_{z}(r, z=d)=2400(1 \pm 0.04) \exp \left[-\left(\frac{r}{9.9^{\prime \prime} \pm 1.0^{\prime \prime}}\right)^{2}\right] \mathrm{G},
\end{gathered}
$$

where $d$ and $H$ are respectively the depth of the magnetic moment measured from the photosphere and the height of the coronal base above the photosphere; $h=2700 \mathrm{~km}$ is the height of the point of occultation observation above the coronal base (see Paper II). Note that $B$ in (1) and (2) refers to the total field strengths while $B_{z}$ in (3) refers to the vertical component only.

We compare our observational results with three theoretical models: (i) a simple dipole model (e.g. Purcell 1981), (ii) the solenoid model of Sakurai \& Uchida (1977), and (iii) Low's (1980) magnetostatic equilibrium model ('M.S.E.' model). All of these models present the cylindrically symmetric fields with no $\phi$-component. We do not consider force-free models (e.g., Nakagawa et al. 1971) in spite of their popular use, since this spot does not appear in a spiral structure as usually predicted by the force-free models.

To constrain model parameters, we first consider the allowable range of the depth, $d$, of the waist of the field (or the location of current coils) that can match the observed magnetic fields at the spot center at three different heights, i.e., $B_{z}(0, d), B(0, d+H)$, and $B(0, d+H+h)$. We then look for the horizontal extent $a$ and the coronal height $H$ that can reproduce the observed radial distribution of the fields, $B_{z}(r, d)$ and $B(r, d+H)$, as closely as possible. We plot in Figure 1 the resulting model values (solid lines) together with our results (shaded area).

For the dipole model, only two parameters need to be specified: the magnetic moment, $m$, and its location, $d$. Therefore, there can be no doubt that the dipole model predicts a more extended field structure at the upper sunspot atmosphere than observed. In the solenoid model, a current coil giving rise to the magnetic moment is allowed to have a finite horizontal extent, $a$, by which we can adjust the field shape outside the coil at a given depth. However, due to the constraint of vertical magnetic fields at three different heights, the choice of $a$ is much limited. The result is that the solenoid model is also not capable of reproducing the confined structure of the coronal fields we observe. The M.S.E. model comes closer to our observation: First of all, it predicts a gaussian form for the field distribution in agreement with our observation (eqs. [1] \& [3]). The horizontal structure of this model is sensitive to the parameter, $\eta$ (see Low 1980 for full expressions), but insensitive to the choice of $H$. This is in a good agreement with our result that the gaussian width changes only a little with height, $H$. The vertical variation is also closely fitted by this model if we locate the waist $(z=0)$ at a depth, $d \simeq 10^{\prime \prime}$. 

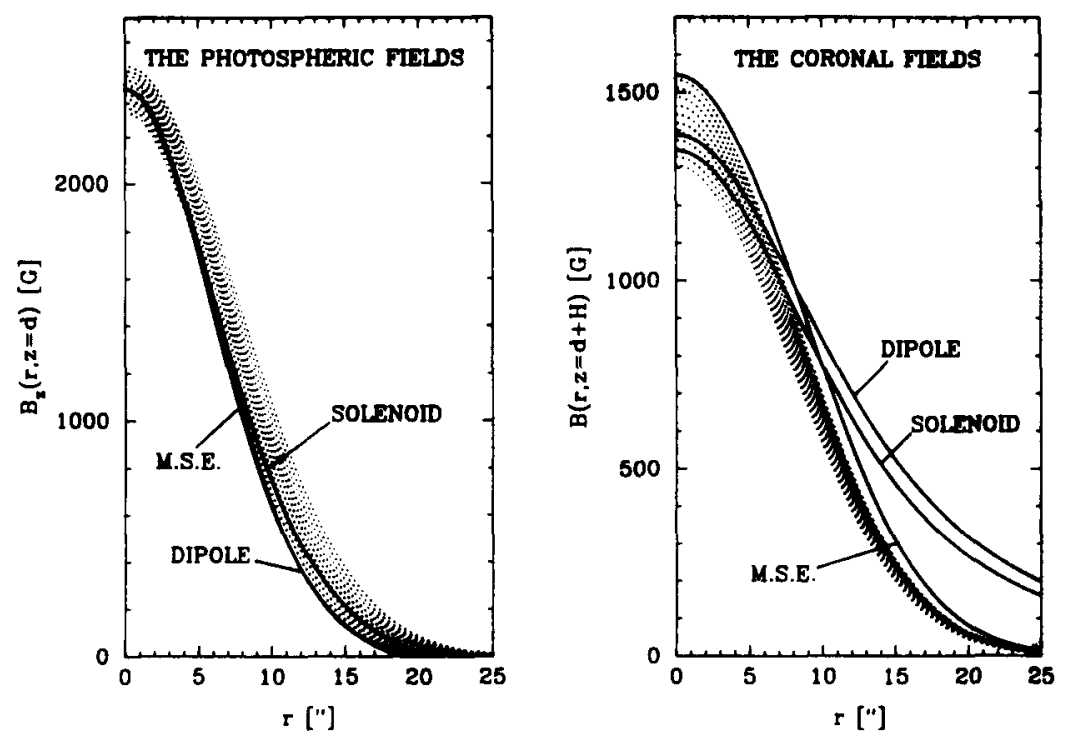

Fig. 1. Comparison of the fields deduced from the observation (shaded area) with theoretical fields (solid lines). Used model parameters are: $d=15^{\prime \prime}$, $H=3.0^{\prime \prime}$, and $m=1.6 \times 10^{30} \mathrm{G} \cdot \mathrm{cm}^{3}$ for the dipole model; $d=13^{\prime \prime}, H=3.0^{\prime \prime}$, $a=4.0^{\prime \prime}$, and $J=1.3 \times 10^{23} \mathrm{esu}^{-\mathrm{sec}^{-1}}$ for the solenoid model; $d=10^{\prime \prime}, H=3.5^{\prime \prime}$, $a=7.0^{\prime \prime}, B_{o}=9.4 \times 10^{20} \mathrm{G} \cdot \mathrm{cm}^{2}$, and $\eta=1.95$ for the M.S.E. model.

\section{DISCUSSION}

The dipole and the solenoid fields are specified solely by source currents in vacuum. In comparison, the M.S.E. model (Low 1980) is based on the exact solution of the magnetohydrostatic equation, which reads

$$
\frac{1}{4 \pi}(\vec{\nabla} \times \vec{B}) \times \vec{B}-\vec{\nabla} p-\rho g \hat{z}=0
$$

where $p$ is the gas pressure, $\rho$ the density, and $g$ the gravitational acceleration. Therefore, the agreement of our observational results to the M.S.E. model would imply that the static interaction between the magnetic curvature force, the pressure gradient, and the gravity serves to shape the sunspot magnetic structure.

The amount of horizontal pressure gradient, $\partial p / \partial r$, or the corresponding pressure difference, $\Delta p=p(r=\infty)-p(r=0)=\int_{0}^{\infty}(\partial p / \partial r) d r$, can be estimated for the magnetic field structure under consideration. We compute the amount of the horizontal pressure difference by using Low's (1980) model together with the parameters found above (Fig. 1). The results are $\Delta p \simeq 1.7 \times 10^{5}$ dyne $/ \mathrm{cm}^{2}$ at the photosphere $(z=d)\left(c f . \Delta p \sim 10^{6}\right.$ dyne $/ \mathrm{cm}^{2}$ adopted by Deinzer 1965 and Yun 1970) and $\Delta p \simeq 0.6 \times 10^{5} \mathrm{dyne} / \mathrm{cm}^{2}$ at the coronal base $(z=d+H)$. Therefore, it can be seen that about one third of the horizontal pressure difference at the photosphere should be maintained at the base of 
the corona in order to make a confined magnetic structure as found in our observations.

The pressure gradient along the vertical direction cannot be assessed without knowledge of the density variation. Along the sunspot axis, however, the magnetic curvature vanishes and thus there are only the vertical pressure gradient and the gravity to be balanced. This explains why even a vacuum model can match the observed vertical field distribution along the sunspot axis. As an aside, it should be noted that the above consideration on the force balance is valid from the photosphere up to the coronal base above which the magnetic field lines could be force-free.

\section{CQNCLUSION}

In this paper, we investigated whether our observational results (Papers I \& II) for the sunspot (NOAA \#4741) coronal fields can be reproduced by any simple theoretical model. It is found that our results can be closely fitted by the M.S.E. model (Low 1980) where static interaction between the magnetic curvature force and the horizontal pressure gradient serves to limit the spread of the field lines with height. We therefore presume that the sunspot fields at the upper atmosphere tends to be kept confined by the pressure gradient across the spot which would otherwise be extended as predicted by the dipole or the solenoid model.

\section{ACKNOWLEDGEMENT}

We thank Dr. B. C. Low for helpful comments. This work was funded by NSF grants ATM-9013173 and AST-8919770 and by NASA grant NAGW-30035 to the California Institute of Technology.

\section{REFERENCES}

Alissandrakis, C. E., Kundu, M. R., \& Lantos, P. 1980 Astr. Ap. 82, 30 Deinzer, M. 1965, Ap. J. 141, 548

Gelfreikh, G. B., Bogod, V. M., Abramov-Maximov, V. E., \& Tsvetkov, S. V. 1992, in H. Zirin (ed.), 'The Magnetic and Velocity Fields of Solar Active Regions', IAU Colloq. No. 141

Hurford, G. J. \& Gary, D. E. 1986, in Coronal Processes and Plasmas, NASA Conference Proc. 2442

Nakagawa, Y., Raadu, M. A., Billings, D. E., \& McNamara, D. 1971, Solar Phys. 19, 72

Lee, J. W., Hurford, G. J., \& Gary, D. E. 1992, Solar Phys. in press (Paper I) Lee, J. W., Gary, D. E., \& Hurford, G. J. 1992, Solar Phys. in press (Paper II) Low, B. C. 1980, Solar Phys. 67, 57

Purcell, E. M. 1981, Electricity and Magnetism, McGraw-Hill, New York Sakurai, K. \& Uchida, Y. 1977, Solar Phys. 52, 397

Yun, H. S. 1970, Ap. J. 162, 975 